\title{
Identifying the Prognosis Factors in Death after Liver Transplantation via Adaptive LASSO in Iran
}

\author{
Hadi Raeisi Shahraki, Saeedeh Pourahmad, and Seyyed Mohammad Taghi Ayatollahi \\ Department of Biostatistics, School of Medicine, Shiraz University of Medical Sciences, Shiraz, Iran \\ Correspondence should be addressed to Seyyed Mohammad Taghi Ayatollahi; ayatolahim@sums.ac.ir
}

Received 28 April 2016; Accepted 7 August 2016

Academic Editor: Pam R. Factor-Litvak

Copyright (C) 2016 Hadi Raeisi Shahraki et al. This is an open access article distributed under the Creative Commons Attribution License, which permits unrestricted use, distribution, and reproduction in any medium, provided the original work is properly cited.

Despite the widespread use of liver transplantation as a routine therapy in liver diseases, the effective factors on its outcomes are still controversial. This study attempted to identify the most effective factors on death after liver transplantation. For this purpose, modified least absolute shrinkage and selection operator (LASSO), called Adaptive LASSO, was utilized. One of the best advantages of this method is considering high number of factors. Therefore, in a historical cohort study from 2008 to 2013, the clinical findings of 680 patients undergoing liver transplant surgery were considered. Ridge and Adaptive LASSO regression methods were then implemented to identify the most effective factors on death. To compare the performance of these two models, receiver operating characteristic (ROC) curve was used. According to the results, 12 factors in Ridge regression and 9 ones in Adaptive LASSO regression were significant. The area under the ROC curve (AUC) of Adaptive LASSO was equal to 89\% (95\% CI: 86\%-91\%), which was significantly greater than Ridge regression $(64 \%, 95 \%$ CI: 61\%-68\%) $(p<0.001)$. As a conclusion, the significant factors and the performance criteria revealed the superiority of Adaptive LASSO method as a penalized model versus traditional regression model in the present study.

\section{Introduction}

Liver transplantation is recognized as a well-established therapy for patients with acute liver failure [1-3]. Despite the fact that it has become widespread and recently the number of liver transplants throughout the world has exceeded 15000 cases in a year, the clinical effective risk factors on liver transplantation outcome are still controversial [4].

Logistic regression is the most common method for assessing the effects of various factors on the binary outcome [5]. Usually, in order to avoid modeling bias, at the initial stage of modeling, a high number of variables are candidates [6]. But logistic regression may encounter with multicollinearity problem (strong correlation between two or more than two independent variables in regression models) in modeling the relation among a high number of variables $[7,8]$. In these settings, Ridge regression is a traditional remedial method which can control multicollinearity by imposing a slight bias in the estimation of coefficients. Penalized regressions have recently developed models in facing high dimensional data.
Imposing a penalty on the coefficients in penalized methods, besides controlling the multicollinearity, represents a sparse and interpretable model [9]. For instance, least absolute shrinkage and selection operator (LASSO), as one of the most famous penalized models, is applicable regardless of the number of variables and sample size [10].

In some of the previous researches in liver disease, penalized methods were applied and superiority of them versus conventional statistical methods was confirmed by some authors $[11,12]$. On the other hand, although the risk of death and their associated effective factors after liver transplantation was investigated in some studies, due to the limitations of conventional statistical methods, a few potential factors were considered. Recently, penalized regression had been widely used in medical sciences for modeling and identifying the most important factors. However, artificial neural networks (ANNs) are frequently used as the nonparametric substituted modeling methods in the issue of large sample size and a high number of variables [2]. 
Therefore, the aim of this study was to identify the prognosis factors in death after liver transplantation among 35 factors, using logistic Ridge regression and logistic Adaptive LASSO which is a modified version of LASSO with weighted penalties [13].

\section{Methods}

Clinical findings of 680 patients undergoing liver transplant surgery were collected in a historical cohort study from 2008 to 2013 at Nemazee Hospital Organ Transplantation Center, Shiraz, southern Iran. Exclusion criteria were transplantation more than once, less than one day of survival, or any kinds of rejection of transplantation. Independent variables (risk factors) included recipient sex, age, weight, diagnosis disease, comorbidity disease, end-stage liver disease (MELD), or pediatric end-stage liver disease (PELD) score, child class, type of transplantation, previous abdominal surgery, renal failure before and after transplantation, diabetes after transplantation, vascular complication after transplantation, primary nonfunction (PNF), posttransplant lymphoproliferative disorder (PTLD), cytomegalovirus (CMV), lung complication after transplantation, bile duct complication after transplantation, exploration after transplantation, child score, waiting list time (day), creatinine, INR, total bilirubin, cold ischemia time (hour), total bleeding (mL), pack cell (bag), duration of operation (hour), duration of hospital stay (day), donor sex, age, and status while the binary dependent variable (response, event) was death due to liver transplantation during the period of five years after the surgery (yes: 1 and no: 0 ).

2.1. Statistical Analyses. In order to identify the most effective factors on death after liver transplantation, we implemented Ridge and Adaptive LASSO regression. In this article, inverse LASSO coefficients were used for each variable as their weight in Adaptive LASSO. The performance of these two models in classification of high and low risk patients was then compared by calculating the areas under the curve (AUC) in receiver operating characteristic (ROC) curve. Statistical analysis was performed using SPSS 20.0, MedCalc 14.0, parcor and ridge packages in R 3.1.3 software.

\section{Results}

The patients' age ranged from 2 to 74 years with a mean (SD) of 33.6 (18.27) including 430 (63.2\%) males and 250 (36.8\%) females. The results revealed that, among 680 patients, only $78(11.47 \%)$ died due to complications from liver transplantation and the others $(88.53 \%)$ were alive. Table 1 shows the qualitative characteristics of recipients and quantitative information is displayed in Table 2.

Considering the final outcome as dependent variable and all 35 mentioned factors in Tables 1 and 2 as independent variable, Ridge regression and Adaptive LASSO regression were fitted and standard error of coefficients was obtained using 500 times bootstrap method. Among these 35 factors, 12 factors in Ridge regression were significant and 9 ones in Adaptive LASSO regression had nonzero coefficient. Table 3
TABLE 1: Descriptive statistics of qualitative variables of 680 patients with liver transplantation utilized in modeling process.

\begin{tabular}{|c|c|}
\hline Characteristic & Number (\%) \\
\hline \multicolumn{2}{|l|}{ Recipient sex } \\
\hline Male & $430(63.2)$ \\
\hline Female & $250(36.8)$ \\
\hline \multicolumn{2}{|c|}{ Recipient diagnosis disease } \\
\hline Metabolic & $102(15)$ \\
\hline Cholestatic & $140(20.6)$ \\
\hline Hepatitis & $267(39.3)$ \\
\hline Tumors & $9(1.3)$ \\
\hline Cryptogenic & $46(6.8)$ \\
\hline Other causes & $116(17.1)$ \\
\hline \multicolumn{2}{|c|}{ Comorbidity disease } \\
\hline No & $573(84.3)$ \\
\hline Yes & $107(15.7)$ \\
\hline \multicolumn{2}{|c|}{ MELD/PELD score } \\
\hline$<20$ & $308(45.3)$ \\
\hline$\geq 20$ & $372(54.7)$ \\
\hline \multicolumn{2}{|l|}{ Child class } \\
\hline A & $81(11.9)$ \\
\hline $\mathrm{B}$ & 305 (44.9) \\
\hline $\mathrm{C}$ & $294(43.2)$ \\
\hline \multicolumn{2}{|c|}{ Type of transplantation } \\
\hline Whole & $573(84.3)$ \\
\hline Split & $36(5.3)$ \\
\hline Partial & $71(10.4)$ \\
\hline \multicolumn{2}{|c|}{ Previous abdominal surgery } \\
\hline No & $590(86.8)$ \\
\hline Yes & $90(13.2)$ \\
\hline \multicolumn{2}{|c|}{ Renal failure before transplantation } \\
\hline No & $646(95)$ \\
\hline Yes & $34(5)$ \\
\hline \multicolumn{2}{|c|}{ Diabetes after transplantation } \\
\hline No & $528(77.6)$ \\
\hline Yes & $152(22.4)$ \\
\hline \multicolumn{2}{|c|}{ Vascular complication after transplantation } \\
\hline No & $646(95)$ \\
\hline Yes & $34(5)$ \\
\hline \multicolumn{2}{|c|}{ Renal failure after transplantation } \\
\hline No & $624(91.8)$ \\
\hline Yes & $56(8.2)$ \\
\hline \multicolumn{2}{|l|}{ PNF } \\
\hline No & $669(98.4)$ \\
\hline Yes & $11(1.6)$ \\
\hline \multicolumn{2}{|l|}{ PTLD } \\
\hline No & $672(98.8)$ \\
\hline Yes & $8(1.2)$ \\
\hline \multicolumn{2}{|l|}{ CMV } \\
\hline No & $651(95.7)$ \\
\hline Yes & $29(4.3)$ \\
\hline \multicolumn{2}{|c|}{ Lung complication after transplantation } \\
\hline No & $655(96.3)$ \\
\hline Yes & $25(3.7)$ \\
\hline
\end{tabular}


TABLE 1: Continued.

\begin{tabular}{lc}
\hline Characteristic & Number $(\%)$ \\
\hline Bile duct complication after transplantation & \\
$\quad$ No & $664(97.6)$ \\
Yes & $16(2.4)$ \\
Exploration after transplantation & \\
$\quad$ No & $567(83.4)$ \\
Yes & $113(16.6)$ \\
Donor sex & \\
Male & $452(66.5)$ \\
Female & $228(33.5)$ \\
Donor status & \\
Living & $70(10.3)$ \\
Died & $610(89.7)$ \\
\hline
\end{tabular}

TABLE 2: Descriptive statistics of quantitative variables of 680 patients with liver transplantation utilized in modeling process.

\begin{tabular}{lc}
\hline Characteristic & Mean (SD) \\
\hline Recipient age (year) & $33.6(18.24)$ \\
Weight (kg) & $58.67(23.30)$ \\
Child score & $9.08(2.18)$ \\
Waiting list time (day) & $167.63(224.87)$ \\
Creatinine (mg/dL) & $0.89(0.59)$ \\
INR & $1.98(1.22)$ \\
Total bilirubin (mg/dL) & $8.14(10.36)$ \\
Cold ischemia time (hour) & $6.76(3.46)$ \\
Total bleeding (mL) & $16.99(1633)$ \\
Pack cell (bag) & $2.40(2.83)$ \\
Fresh frozen plasma (bag) & $3.19(4.01)$ \\
Duration of operation (hour) & $6.03(1.28)$ \\
Duration of hospital stay (day) & $12.84(7.73)$ \\
Donor age (year) & $31.2(15.25)$ \\
\hline
\end{tabular}

represents the results of fitting these two models and Figure 1 displays the coefficients of Adaptive LASSO regression for each factor in bootstrap method. In order to compare the performances of these two models, the values of $p_{i}$ (risk of death due to complications from liver transplantation) were calculated using the reported coefficients in Table 3 for each model and then patients with high and low risk of death were classified using optimal cut-off point in ROC curve.

Adaptive LASSO revealed that the risk of death after transplantation in patients with PNF was 1.75 -fold compared to the others. Also, renal failure after transplantation with relative risk of 1.42 , lung complication after transplantation with relative risk of 1.26 , and PTLD with relative risk of 1.23 were introduced as the most important risk factors which can increase the risk of death (Table 3). The AUC of our proposed penalized model was equal to $89 \%$ (95\% CI: $86 \%-91 \%$ ), which was significantly greater than Ridge regression $(68 \%, 95 \%$ CI: $64 \%-71 \%)(p<0.001)$. Also, sensitivity and specificity for Adaptive LASSO were $82 \%$ and $86 \%$, respectively (Table 4 ). Figure 2 compares the ROC curves of two utilized models.

\section{Discussion}

Clinical findings of 680 patients undergoing liver transplant surgery by two considered methods revealed that PNF, PTLD, renal failure, and lung complication after transplantation were the most important prognosis factors in death after liver transplantation with different coefficients or relative risks (Table 3).

Although Ridge regression is known as an effective remedial method for controlling multicollinearity, large values of standard errors and unrealistic coefficients in the second and third columns of Table 3 confirm the presence of multicollinearity in this model. On the other hand, sufficiently small values of standard errors of coefficients in Adaptive LASSO demonstrate controlling the multicollinearity. By omitting the redundant factors, Adaptive LASSO could estimate the risk of death much better than traditional logistic Ridge regression (Table 4). The current study showed that Adaptive LASSO, as a penalized regression, was better than the Ridge regression model in predicting the risk of death in patients after liver transplantation based on the area under the ROC curve (Figure 2 and Table 4).

In our study, PNF was the most important prognosis factor in death after transplantation; this result is in agreement with most of the previous studies about survival after transplantation or risk factors of death after transplantation [14]. It may be due to direct association between PNF and complexity of the surgical procedure that can increase the risk of death [15].

Recently, several studies about the role of renal failure, as an important prognosis factor in death, in hepatitis and kidney disease have been performed [16, 17]. Also, the association of renal failure with increase of morbidity and mortality after heart or liver transplantation in some studies was approved; this is consistent with our findings $[18,19]$. In addition, lung and vascular complication after transplantation in our proposed models were represented as two effective factors in death which are considered as two common and main causes of death after liver transplantation [20-22]. In a study about complications and mortality after liver transplantation, both univariate and multivariate analyses revealed a significant association between vascular complication and mortality of recipients [23]. As another result of the current study, like the other studies, we found that PTLD can increase the risk of death [24, 25]. And finally in contrast to our study, factors like the recipient's age, comorbidity disease, previous abdominal surgery, and MELD were reported as death risk factors after transplantation [2, $14,26]$. As a limitation of this study, we can refer to the low number of death (11\%) due to the nature of the data.

\section{Conclusion}

To the best of our knowledge, our study was one of the first researches in Iran which considered simultaneous effect of 35 potential factors on the risk of death after transplantation by two powerful statistical methods. Adaptive LASSO regression demonstrates superiority of penalized models versus traditional regression models in facing a high number of variables. 
TABLE 3: Coefficients of nonzero factors in Ridge and Adaptive LASSO logistic regression.

\begin{tabular}{|c|c|c|c|c|c|}
\hline \multirow{3}{*}{ Characteristic } & \multicolumn{5}{|c|}{ Method } \\
\hline & \multicolumn{2}{|c|}{ Ridge regression } & \multicolumn{3}{|c|}{ Adaptive LASSO regression } \\
\hline & Coefficient & SE & Coefficient & $\mathrm{SE}$ & $\mathrm{RR}$ \\
\hline PNF & 8.51 & 1.98 & 0.56 & 0.10 & 1.75 \\
\hline Renal failure after transplantation & 13.59 & 1.86 & 0.35 & 0.07 & 1.42 \\
\hline Lung complication after transplantation & 6.42 & 1.85 & 0.23 & 0.10 & 1.26 \\
\hline PTLD & 3.70 & 1.74 & 0.21 & 0.22 & 1.23 \\
\hline Vascular complication after transplantation & 6.49 & 1.80 & 0.17 & 0.10 & 1.19 \\
\hline Exploration after transplantation & 9.93 & 1.92 & 0.14 & 0.06 & 1.15 \\
\hline \multicolumn{6}{|l|}{ Type of transplantation } \\
\hline Whole & Baseline & - & Baseline & - & - \\
\hline Split & 4.06 & 1.89 & 0.00 & 0.06 & 1.00 \\
\hline Partial & 2.96 & 1.26 & 0.03 & 0.15 & 1.03 \\
\hline Duration of operation (hour) & 6.31 & 1.97 & 0.02 & 0.01 & 1.02 \\
\hline \multicolumn{6}{|l|}{ Donor sex } \\
\hline Male & Baseline & - & Baseline & - & - \\
\hline Female & 4.25 & 2.03 & 0.02 & 0.02 & 1.02 \\
\hline Donor age & 4.45 & 1.93 & 0.00 & 0.01 & 1.00 \\
\hline Diabetes after transplantation & -4.02 & 1.89 & 0.00 & 0.03 & 1.00 \\
\hline
\end{tabular}

TABLE 4: Sensitivity, specificity, and AUC of Ridge regression and Adaptive LASSO methods in modeling effective factors on death of 680 patients after liver transplantation.

\begin{tabular}{lccr}
\hline Method & AUC (95\% CI) & Sensitivity (95\% CI) & Specificity (95\% CI) \\
\hline Ridge & $67.7(64-71.2)$ & $84.6(74.7-91.8)$ & $46.7(42.6-50.8)$ \\
Adaptive LASSO & $89(86.4-91.2)$ & $82.1(71.7-89.8)$ & $85.5(82.5-88.3)$ \\
\hline
\end{tabular}

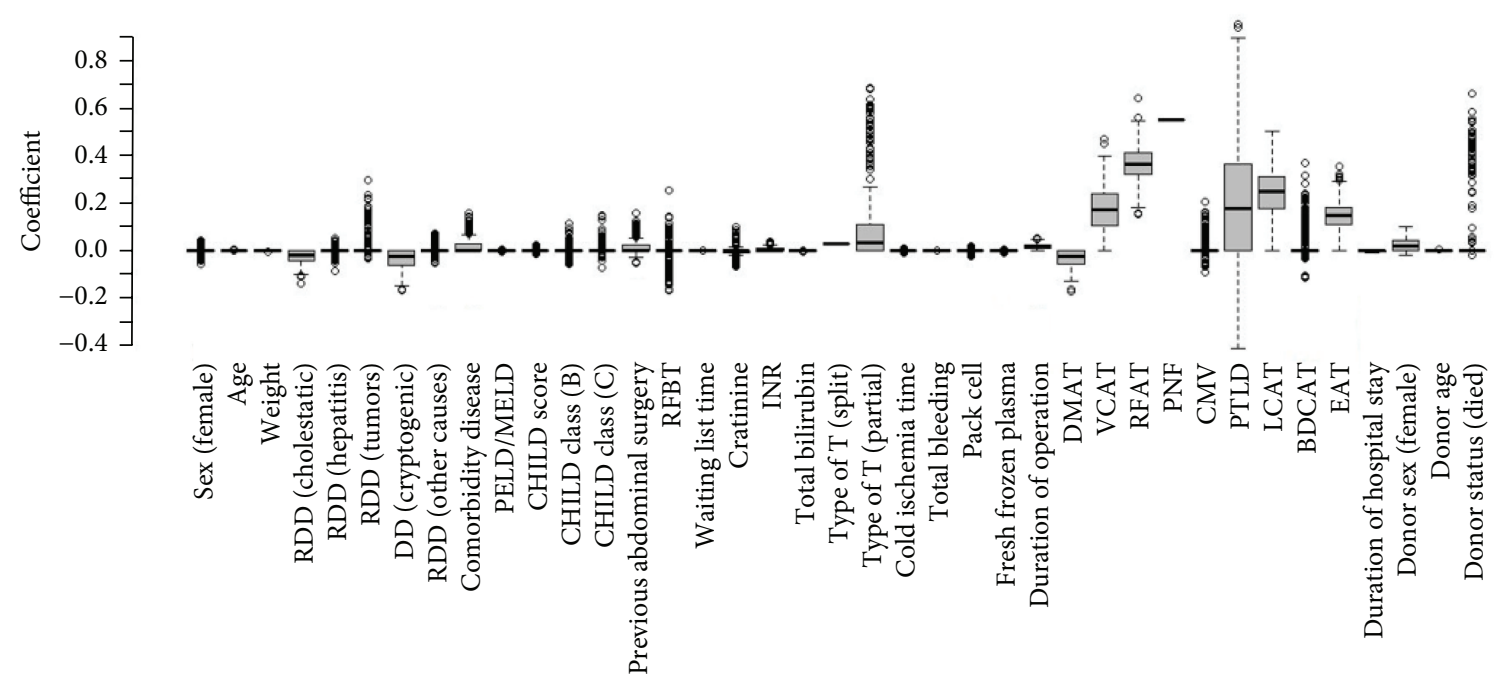

FIGURE 1: The coefficients of Adaptive LASSO in 500 times bootstrap method. RDD: recipient diagnosis disease, MELD: model for endstage liver disease, PELD: pediatric end-stage liver disease, RFBT: renal failure before transplantation, DMAT: diabetes mellitus after transplantation, VCAT: vascular complication after transplantation, RFAT: renal failure after transplantation, PNF: primary nonfunction, CMV: cytomegalovirus, PTLD: posttransplant lymphoproliferative disorder, LCAT: lung complication after transplantation, BDCAT: bile duct complication after transplantation, EAT: exploration after transplantation. 


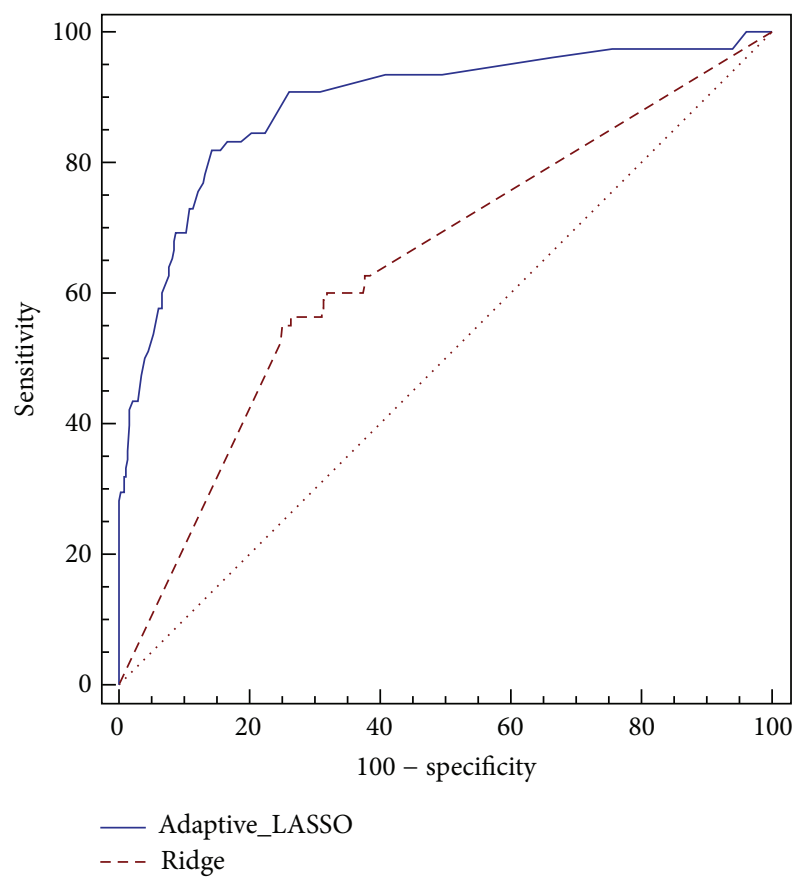

FIgure 2: The area under the ROC curve for Ridge and Adaptive LASSO models.

\section{Competing Interests}

There was no conflict of interests regarding the publication of this paper.

\section{Acknowledgments}

The authors are thankful to Miss Bahareh Khosravi and the Nemazee Hospital Organ Transplant Center, Shiraz, Iran, for their help in data gathering. Also, authors would like to thank Center for Development of Clinical Research of Nemazee Hospital and Dr. Nasrin Shokrpour for editorial assistance.

\section{References}

[1] R. Adam, V. Cailliez, P. Majno et al., "Normalised intrinsic mortality risk in liver transplantation: European Liver Transplant Registry study," The Lancet, vol. 356, no. 9230, pp. 621-627, 2000.

[2] B. Khosravi, S. Pourahmad, A. Bahreini, S. Nikeghbalian, and G. Mehrdad, "Five years survival of patients after liver transplantation and its effective factors by neural network and cox poroportional hazard regression models," Hepatitis Monthly, vol. 15, no. 9, 2015.

[3] P. R. Salvalaggio, J. C. Caicedo, L. C. de Albuquerque et al., "Liver transplantation in Latin America: the state-of-the-art and future trends," Transplantation, vol. 98, no. 3, pp. 241-246, 2014.

[4] I. H. Matinlauri, M. M. Nurminen, K. A. Höckerstedt, and H. M. Isoniemi, "Risk factors predicting survival of liver transplantation," Transplantation Proceedings, vol. 37, no. 2, pp. 1155-1160, 2005.

[5] J. D. Geller, N. Zuckerman, and A. Seidel, "Service-learning as a catalyst for community development: how do community partners benefit from service-learning?" Education and Urban Society, vol. 48, no. 2, pp. 151-175, 2016.

[6] J. Fan and R. Li, "Variable selection via nonconcave penalized likelihood and its oracle properties," Journal of the American Statistical Association, vol. 96, no. 456, pp. 1348-1360, 2001.

[7] A. M. Aguilera, M. Escabias, and M. J. Valderrama, "Using principal components for estimating logistic regression with high-dimensional multicollinear data," Computational Statistics and Data Analysis, vol. 50, no. 8, pp. 1905-1924, 2006.

[8] I. I. Spyroglou, E. A. Chatzimichail, E. Spanou, E. Paraskakis, and A. G. Rigas, "Ridge regression and bootstrapping in asthma prediction," in Proceedings of the Mathematical Methods, Mathematical Models and Simulation in Science and Engineering Conference, Vienna, Austria, 2005.

[9] H. R. Shahraki, A. Salehi, and N. Zare, "Survival prognostic factors of male breast cancer in Southern Iran: a LASSOCox regression approach," Asian Pacific Journal of Cancer Prevention, vol. 16, no. 15, pp. 6773-6777, 2015.

[10] R. Tibshirani, "Regression shrinkage and selection via the lasso," Journal of the Royal Statistical Society, Series B: Methodological, vol. 58, no. 1, pp. 267-288, 1996.

[11] K. E. Corey, U. Kartoun, H. Zheng, and S. Y. Shaw, "Development and validation of an algorithm to identify nonalcoholic fatty liver disease in the electronic medical record," Digestive Diseases and Sciences, vol. 61, no. 3, pp. 913-919, 2016.

[12] K. England, C. Thorne, L. Pembrey, P.-A. Tovo, and M.L. Newell, "Age-and sex-related reference ranges of alanine aminotransferase levels in children: European paediatric HCV Network," Journal of Pediatric Gastroenterology and Nutrition, vol. 49, no. 1, pp. 71-77, 2009.

[13] H. Zou, "The adaptive lasso and its oracle properties," Journal of the American Statistical Association, vol. 101, no. 476, pp. 1418$1429,2006$.

[14] Z. Máthé, A. Paul, E. P. Molmenti et al., "Liver transplantation with donors over the expected lifespan in the model for endstaged liver disease era: is Mother Nature punishing us?" Liver International, vol. 31, no. 7, pp. 1054-1061, 2011.

[15] R. Sharma, R. Kashyap, A. Jain et al., "Surgical complications following liver transplantation in patients with portal vein thrombosis-a single-center perspective," Journal of Gastrointestinal Surgery, vol. 14, no. 3, pp. 520-527, 2010.

[16] R. Luo, A. Greenberg, and C. D. Stone, "Increasing volume but decreasing mortality of hospitalized hepatitis $\mathrm{C}$ patients in the United States, 2005 to 2011," Journal of Clinical Gastroenterology, vol. 49, no. 7, pp. 620-627, 2015.

[17] M. Scarpino, M. R. Pinzone, M. Di Rosa et al., "Kidney disease in HIV-infected patients," European Review for Medical and Pharmacological Sciences, vol. 17, no. 19, pp. 2660-2667, 2013.

[18] F. Tinti, A. P. Mitterhofer, and P. Muiesan, "Liver transplantation: role of immunosuppression, renal dysfunction and cardiovascular risk factors," Minerva Chirurgica, vol. 67, no. 1, pp. 1-13, 2012.

[19] C. A. Q. Santos, D. C. Brennan, V. J. Fraser, and M. A. Olsen, "Incidence, risk factors, and outcomes of delayed-onset cytomegalovirus disease in a large, retrospective cohort of heart transplant recipients," Transplantation Proceedings, vol. 46, no. 10, pp. 3585-3592, 2014.

[20] M. B. Fallon, M. J. Krowka, R. S. Brown et al., "Impact of hepatopulmonary syndrome on quality of life and survival in liver transplant candidates," Gastroenterology, vol. 135, no. 4, pp. $1168-1175,2008$. 
[21] S. Yilmaz, C. Kayaalp, C. Ara et al., "Single-center analysis of the first 304 living-donor liver transplantations in 3 years," HepatoGastroenterology, vol. 60, no. 125, pp. 1105-1109, 2013.

[22] Y.-L. Yang and Z.-W. Liu, "Analysis on biliary complications after orthotopic liver transplantation using biliary tract endoscopy," Journal of Clinical Rehabilitative Tissue Engineering Research, vol. 14, no. 18, pp. 3417-3420, 2010.

[23] E. H. Gad, A. Alsebaey, M. Lotfy, M. Eltabbakh, and A. A. Sherif, "Complications and mortality after adult to adult living donor liver transplantation: a retrospective cohort study," Annals of Medicine and Surgery, vol. 4, no. 2, pp. 162-171, 2015.

[24] V. R. Dharnidharka, K. L. Martz, D. M. Stablein, and M. R. Benfield, "Improved survival with recent post-transplant lymphoproliferative disorder (PTLD) in children with kidney transplants," American Journal of Transplantation, vol. 11, no. 4, pp. 751-758, 2011.

[25] A. Dreyzin, J. Lunz, V. Venkat et al., "Long-term outcomes and predictors in pediatric liver retransplantation," Pediatric Transplantation, vol. 19, no. 8, pp. 866-874, 2015.

[26] P. Chen, W. Wang, L. Yan et al., "Risk factors for firstyear hospital readmission after liver transplantation," European Journal of Gastroenterology \& Hepatology, vol. 27, no. 5, pp. 600606, 2015. 


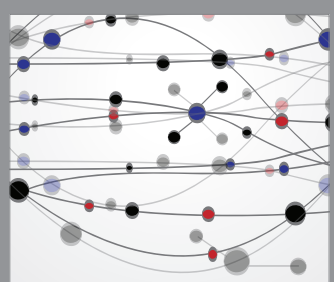

The Scientific World Journal
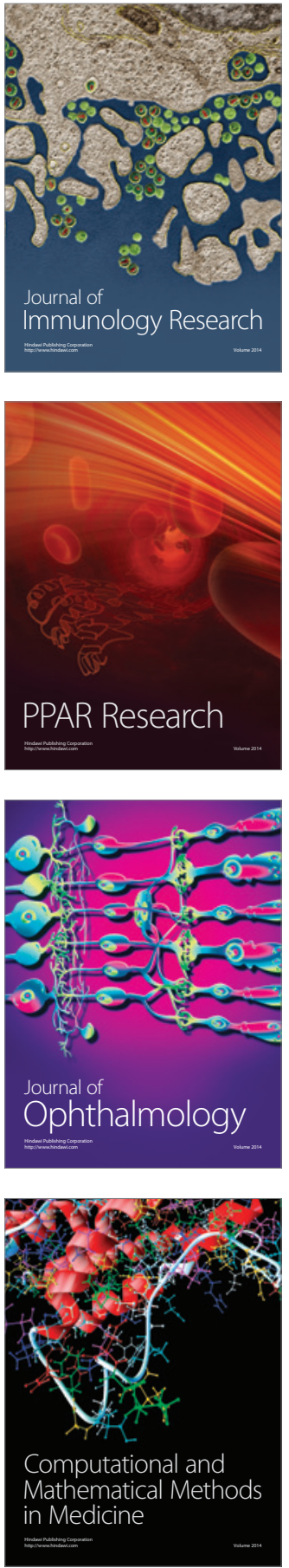

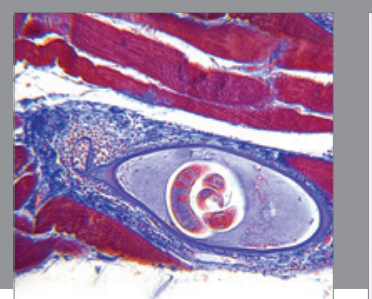

Gastroenterology Research and Practice

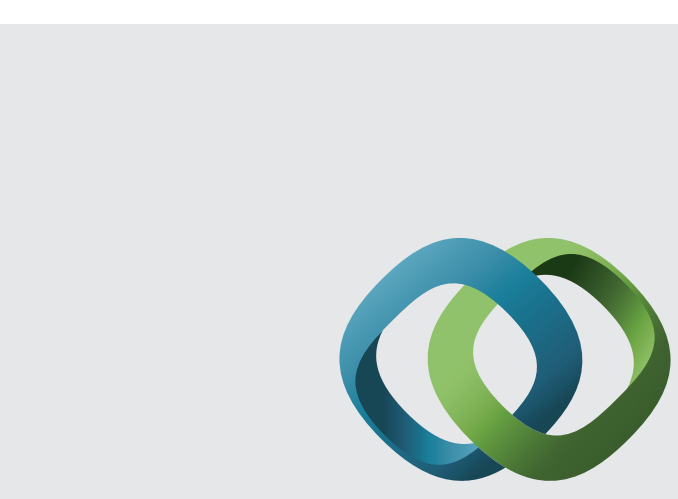

\section{Hindawi}

Submit your manuscripts at

http://www.hindawi.com
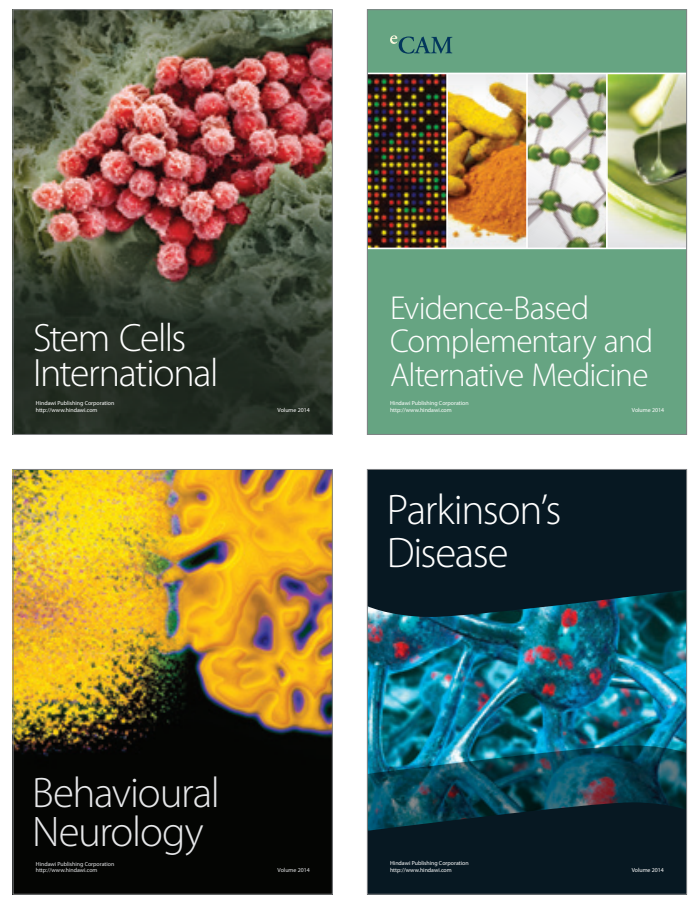
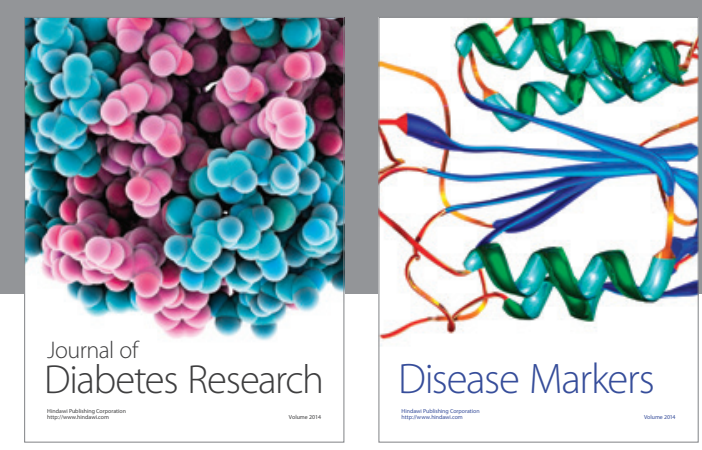

Disease Markers
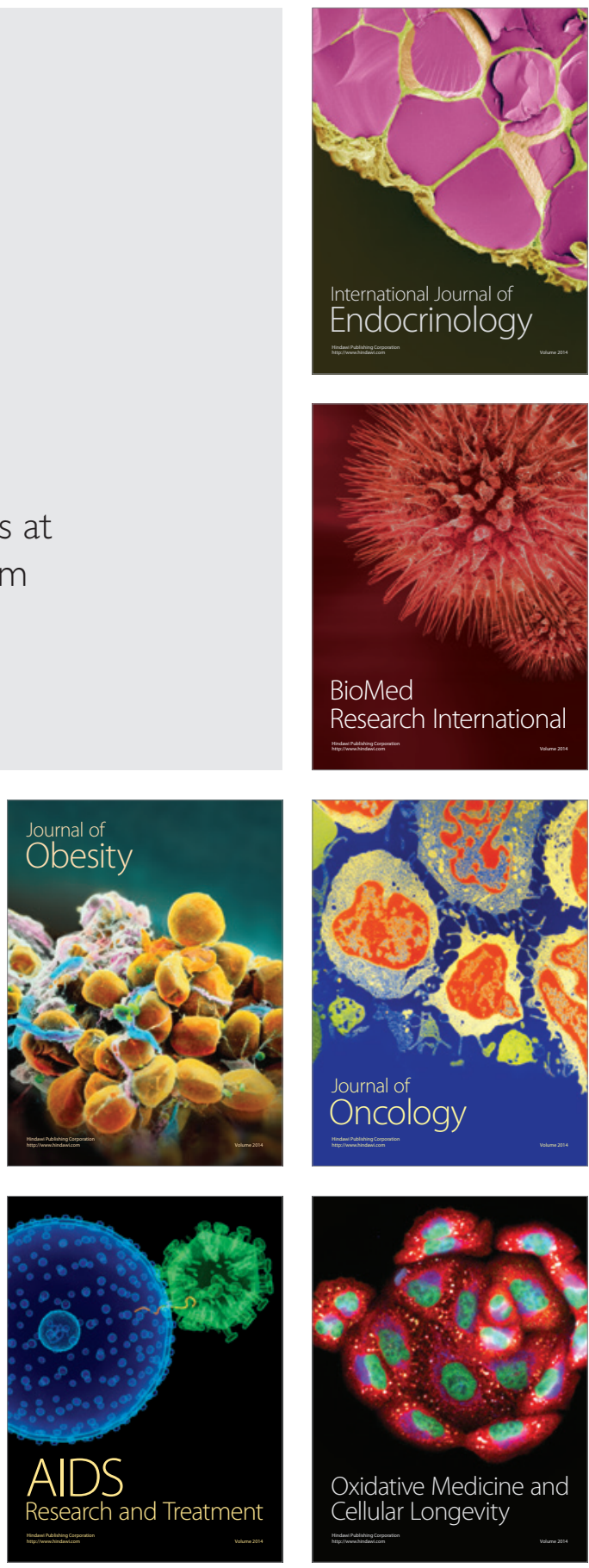